\title{
Microfluidic Production of Biopolymer Microcapsules with
}

\section{Controlled Morphology}

\section{Supporting Information}

Hong Zhang, ${ }^{\dagger}$ Ethan Tumarkin, ${ }^{\dagger}$ Raheem Peerani, ${ }^{\dagger}{ }^{\dagger}$ Zhihong Nie, ${ }^{\dagger}$ Ruby May A. Sullan, ${ }^{\dagger}$ Gilbert C. Walker, ${ }^{\dagger}$ and Eugenia Kumacheva ${ }^{*}, \dagger, \ddagger, \S$

${ }^{\dagger}$ Department of Chemistry, University of Toronto, 80 Saint George Street, Toronto, Ontario M5S 3H6, Canada

${ }^{\ddagger}$ Institute of Biomaterials and Biomedical Engineering, Department of Materials Science and Engineering, University of Toronto, 4 Taddle Creek Road, Toronto, Ontario M5S 3G9, Canada,

${ }^{\S}$ Department of Chemical Engineering and Applied Chemistry, University of Toronto, 200 College Street, Toronto, Ontario M5S 3E5, Canada

Email: ekumache@chem.utoronto.ca 


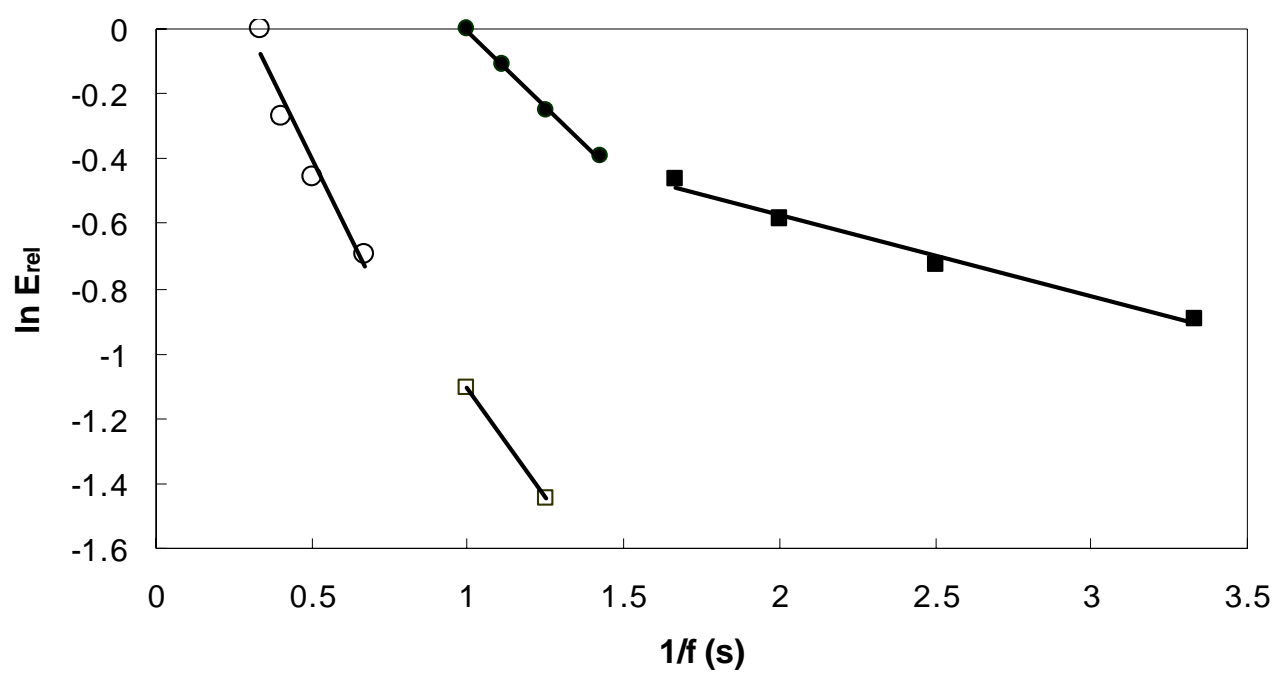

Figure S1. Natural logarithm of relative dissipated energy for alginate capsule (filled) and particle (open) versus cycle time of AFM tip. Elasticity relaxation times of the microgels are obtained from the slopes of these lines, $\tau=(\text { slope })^{-1}$. The primary conclusion is that the relaxation times of the samples are similar. The elasticity relaxation is obtained by examining the force difference between the approach and retrace curves at a given value of the indentation. 